\title{
On the Complexity of Semantic Equivalences for Pushdown Automata and BPA
}

\author{
Antonín Kučera ${ }^{\star 1}$ and Richard Mayr ${ }^{2}$ \\ ${ }^{1}$ Faculty of Informatics, Masaryk University, Botanická 68a, 60200 Brno, Czech Republic, \\ tony@fi.muni.cz \\ 2 Department of Computer Science, Albert-Ludwigs-University Freiburg \\ Georges-Koehler-Allee 51, D-79110 Freiburg, Germany. \\ mayrri@informatik.uni-freiburg.de
}

\begin{abstract}
We study the complexity of comparing pushdown automata (PDA) and context-free processes (BPA) to finite-state systems, w.r.t. strong and weak simulation preorder/equivalence and strong and weak bisimulation equivalence. We present a complete picture of the complexity of all these problems. In particular, we show that strong and weak simulation preorder (and hence simulation equivalence) is EXPTIME-complete between PDA/BPA and finite-state systems in both directions. For PDA the lower bound even holds if the finite-state system is fixed, while simulation-checking between BPA and any fixed finitestate system is already polynomial. Furthermore, we show that weak (and strong) bisimilarity between PDA and finite-state systems is PSPACE-complete, while strong (and weak) bisimilarity between two PDAs is EXPTIME-hard.
\end{abstract}

\section{Introduction}

Transition systems are a fundamental and widely accepted model of processes with discrete states and dynamics (such as computer programs). Formally, a transition system is a triple $\mathcal{T}=(S, A c t, \rightarrow)$ where $S$ is a set of states (or processes), Act is a finite set of actions, and $\rightarrow \subseteq S \times A c t \times S$ is a transition relation. We write $s \stackrel{a}{\rightarrow} t$ instead of $(s, a, t) \in \rightarrow$ and we extend this notation to elements of $A c t^{*}$ in the natural way. A state $t$ is reachable from a state $s$, written $s \rightarrow^{*} t$, iff $s \stackrel{w}{\rightarrow} t$ for some $w \in A c t^{*}$.

In the equivalence-checking approach to formal verification, one describes the specification (the intended behavior) and the actual implementation of a given process as states in transition systems, and then it is shown that they are equivalent. Here the notion of equivalence can be formalized in various ways according to specific needs of a given practical problem (see, e.g., [15] for an overview). It seems, however, that simulation and bisimulation equivalence are of special importance as their accompanying theory has been developed very intensively and found its way to many practical applications. Let $\mathcal{T}=(S, A c t, \rightarrow)$ be a transition system. A binary relation $R \subseteq S \times S$ is a simulation iff whenever $(s, t) \in R$, then for each $s \stackrel{a}{\rightarrow} s^{\prime}$ there is some $t \stackrel{a}{\rightarrow} t^{\prime}$ such that $\left(s^{\prime}, t^{\prime}\right) \in R$. A process $s$ is simulated by $t$, written $s \sqsubseteq t$, iff there is a simulation $R$ such

\footnotetext{
${ }^{\star}$ Supported by the Grant Agency of the Czech Republic, grant No. 201/00/0400.
} 
that $(s, t) \in R$. Processes $s, t$ are simulation equivalent, written $s \simeq t$, iff they can simulate each other. A bisimulation is a symmetric simulation relation, and two processes $s$ and $t$ are bisimilar iff they are related by some bisimulation. In order to abstract from internal ('invisible') transitions of a given system, simulations and bisimulations are sometimes considered in their weak forms. Here, the silent steps are usually modeled by a distinguished action $\tau$, and the extended transition relation $\Rightarrow \subseteq S \times A c t \times S$ is defined by $s \stackrel{a}{\Rightarrow} t$ iff either $s=t$ and $a=\tau$, or $s \stackrel{\tau^{i}}{\rightarrow} s^{\prime} \stackrel{a}{\rightarrow} t^{\prime} \stackrel{\tau^{j}}{\rightarrow} t$ for some $i, j \in \mathbb{N}_{0}$ and $s^{\prime}, t^{\prime} \in S$.

Simulations (and bisimulations) can also be viewed as games [12,14] between two players, the attacker and the defender. In a simulation game the attacker wants to show that $s \nsubseteq t$, while the defender attempts to frustrate this. Imagine that there are two tokens put on states $s$ and $t$. Now the two players, attacker and defender, start to play a simulation game which consists of a (possibly infinite) number of rounds where each round is performed as follows: The attacker takes the token which was put on $s$ originally and moves it along a transition labeled by (some) $a$; the task of the defender is to move the other token along a transition with the same label. If one player cannot move then the other player wins. The defender wins every infinite game. It can be easily shown that $s \sqsubseteq t$ iff the defender has a universal winning strategy. The only difference between a simulation game and a bisimulation game is that the attacker can choose his token at the beginning of every round (the defender has to respond by moving the other token). Again we get that $s \sim t$ iff the defender has a winning strategy. Corresponding 'weak forms' of the two games are defined in the obvious way. We use the introduced games at some points to give a more intuitive justification for our claims. Simulations and bisimulations can also be used to relate states of different transition systems; formally, two systems are considered to be a single one by taking the disjoint union.

In this paper we mainly consider processes of pushdown automata, which are interpreted as a (natural) model of sequential systems with mutually recursive procedures. A pushdown automaton is a tuple $\Delta=(Q, \Gamma, A c t, \delta)$ where $Q$ is a finite set of control states, $\Gamma$ is a finite stack alphabet, Act is a finite input alphabet, and $\delta:(Q \times \Gamma) \rightarrow \mathcal{P}\left(\right.$ Act $\left.\times\left(Q \times \Gamma^{*}\right)\right)$ is a transition function with finite image (here $\mathcal{P}(M)$ denotes the power set of $M)$. We can assume (w.l.o.g.) that each transition increases the height (or length) of the stack by at most one (each PDA can be efficiently transformed to this kind of normal form). In the rest of this paper we adopt a more intuitive notation, writing $p A \stackrel{a}{\rightarrow} q \beta \in \delta$ instead of $(a,(q, \beta)) \in \delta(p, A)$. To $\Delta$ we associate the transition system $\mathcal{T}_{\Delta}$ where $Q \times \Gamma^{*}$ is the set of states (we write $p \alpha$ instead of $(p, \alpha))$, Act is the set of actions, and the transition relation is determined by $p A \alpha \stackrel{a}{\rightarrow} q \beta \alpha$ iff $p A \stackrel{a}{\rightarrow} q \beta \in \delta$.

Let $A, B$ be classes of processes. The problem whether a given process $s$ of $A$ is simulated (or weakly simulated) by a given process $t$ of $B$ is denoted by $A \sqsubseteq B$ (or $A \sqsubseteq w B$, respectively). Similarly, the problem if $s$ and $t$ are simulation equivalent, weakly simulation equivalent, bisimilar, or weakly bisimilar, is denoted by $A \simeq B$, $A \simeq_{w} B, A \sim B$, or $A \approx B$, respectively. The classes of all pushdown processes and finite-state processes (i.e., processes of finite-state transition systems) are denoted PDA and FS, respectively. BPA (basic process algebra), also called context-free processes, is the subclass of PDA where $|Q|=1$, i.e., without a finite-control. 
The state of the art for simulation:

It has been known for some time that strong simulation preorder between PDA and FS is decidable in exponential time. This is because one can reduce the simulation problem to the model-checking problem with PDA and a fixed formula of the modal $\mu$-calculus (see, e.g., [6, 4]). As model checking PDA with the modal $\mu$-calculus is EXPTIMEcomplete [17] the result follows. A PSPACE lower bound for the FS $\sqsubseteq$ BPA problem and a co- $\mathcal{N} \mathcal{P}$ lower bound for the BPA $\sqsubseteq$ FS and BPA $\simeq$ FS problems have been shown in [6]. Furthermore, an $\operatorname{EXPTIME}$ lower bound for the FS $\sqsubseteq$ PDA and $\mathbf{F S} \simeq$ PDA problems have been shown in [4], but in these constructions the finite-state systems were not fixed. The problems of comparing two different BPA/PDA processes w.r.t. simulation preorder/equivalence are all undecidable.

Our contribution:

We show that the problems BPA $\sqsubseteq$ FS, FS $\sqsubseteq$ BPA and BPA $\simeq$ FS are EXPTIMEcomplete, but polynomial for every fixed finite-state system. On the other hand, the problems PDA $\sqsubseteq \mathbf{F S}, \mathbf{F S} \sqsubseteq \mathbf{P D A}$ and $\mathbf{P D A} \simeq \mathbf{F S}$ are $E X P T I M E$-complete, even for a fixed finite-state system. Here, the main point are the lower bounds, which require some new insights into the power of the defender in simulation games. The matching upper bounds are obtained by a straightforward extension of the above mentioned reduction to the model-checking problem with the modal $\mu$-calculus.

\section{The state of the art for bisimulation:}

It was known that strong and weak bisimulation equivalence between PDA and FS is decidable in exponential time, because one can construct (in polynomial time) characteristic modal $\mu$-calculus formulae for the finite-state system and thus reduce the problem to model checking the PDA with a modal $\mu$-calculus formula [11], which is decidable in exponential time [17]. The best known lower bound for the PDA $\approx$ FS problem was PSPACE-hardness, which even holds for a fixed finite state system [8]. The problem PDA $\sim$ FS is also PSPACE-hard, but polynomial in the size of the PDA for every fixed finite-state system [8]. Interestingly, the problem BPA $\approx$ FS (and BPA $\sim$ FS) is polynomial [7]. The symmetric problem of PDA $\sim$ PDA is decidable $[9,13]$, but the complexity is not known. So far, the best known lower bound for it was PSPACE-hardness [8]. The decidability of the PDA $\approx$ PDA problem is still open.

\section{Our contribution:}

We show that the problems PDA $\sim$ FS and PDA $\approx$ FS are PSPACE-complete by improving the known EXPTIME upper bound to PSPACE. Furthermore, we show that the symmetric problem PDA $\sim$ PDA is EXPTIME-hard, by improving the known PSPACE lower bound to EXPTIME. This new EXPTIME lower bound even holds for the subclass of normed PDA.

Due to space constraints, several proofs are omitted. They can be found in the full version of the paper [5].

\section{Lower Bounds}

In this section we prove that all of the problems BPA $\sqsubseteq$ FS, FS $\sqsubseteq$ BPA and BPA $\simeq$ FS are EXPTIME-hard. The problems PDA $\sqsubseteq$ FS, FS $\sqsubseteq$ PDA, PDA $\simeq$ FS are EXPTIME-hard even for a fixed finite-state system. Moreover, we show EXPTIMEhardness of the PDA $\sim$ PDA problem. 
An alternating $L B A$ is a tuple $\mathcal{M}=\left(S, \Sigma, \gamma, s_{0}, \vdash, \dashv, \pi\right)$ where $S, \Sigma, \gamma, s_{0}, \vdash$, and $\dashv$ are defined as for ordinary non-deterministic LBA. In particular, $S$ is a finite set of control states (we reserve ' $Q$ ' to denote a set of control states of pushdown automata), $\vdash, \dashv \in \Sigma$ are the left-end and right-end markers, respectively, and $\pi: S \rightarrow\{\forall, \exists, a c c, r e j\}$ is a function which partitions the control states of $S$ into universal, existential, accepting, and rejecting, respectively. We assume (w.l.o.g.) that $\gamma$ is defined so that

- for all $s \in S$ and $A \in \Sigma$ such that $\pi(s)=\forall$ or $\pi(s)=\exists$ we have that $|\gamma(s, A)|=2$ (i.e., $\gamma(s, A)=\left\{s_{1}, s_{2}\right\}$ for some $\left.s_{1}, s_{2} \in S\right)$. The first element of $\gamma(s, A)$ is denoted by $\operatorname{first}(s, A)$, and the second one by $\operatorname{second}(s, A)$. It means that each configuration of $\mathcal{M}$ where the control state is universal or existential has exactly two immediate successors (configurations reachable in one computational step).

- for all $s \in S$ and $A \in \Sigma$ such that $\pi(s)=a c c$ or $\pi(s)=r e j$ we have that $\gamma(s, A)=\emptyset$, i.e., each configuration of $\mathcal{M}$ where the control state is accepting or rejecting is 'terminated' (without any successors).

A computational tree for $\mathcal{M}$ on a word $w \in \Sigma^{*}$ is a finite tree $T$ satisfying the following: the root of $T$ is (labeled by) the initial configuration $s_{0} \vdash w \dashv$ of $\mathcal{M}$, and if $N$ is a node of $\mathcal{M}$ labeled by a configuration $u s v$ where $u, v \in \Sigma^{*}$ and $s \in S$, then the following holds:

- if $s$ is accepting or rejecting, then $T$ is a leaf;

- if $s$ is existential, then $T$ has one successor whose label is one of the two configurations reachable from $u s v$ in one step (here, the notion of a computational step is defined in the same way as for 'ordinary' Turing machines);

- if $s$ is universal, then $T$ has two successors labeled by the two configurations reachable from $u s v$ in one step.

$\mathcal{M}$ accepts $w$ iff there is a computational tree $T$ such that all leafs of $T$ are accepting configurations. The acceptance problem for alternating LBA is known to be EXPTIME-complete.

In subsequent proofs we often use $M_{\star}$ to denote the set $M \cup\{\star\}$ where $M$ is a set and $\star \notin M$ is a fresh symbol.

\section{Theorem 1. The problem BPA $5 \boldsymbol{F S}$ is EXPTIME-hard.}

Proof. Let $\mathcal{M}=\left(S, \Sigma, \gamma, s_{0}, \vdash, \dashv, \pi\right)$ be an alternating LBA and $w \in \Sigma^{*}$ an input word. We construct (in polynomial time) a BPA system $\Delta=(\Gamma, A c t, \delta)$, a finitestate system $\mathcal{F}=(S, A c t, \rightarrow)$, and processes $\alpha$ and $X$ of $\Delta$ and $\mathcal{F}$, resp., such that $\mathcal{M}$ accepts $w$ iff $\alpha \nsubseteq X$. Let $n$ be the length of $w$. We put $\Gamma=S_{\star} \times \Sigma \cup$ $S \times \Sigma_{\star} \times\{0, \cdots, n+2\} \cup S \times \Sigma \times\{W\} \cup\{T, Z\}$. Configurations of $\mathcal{M}$ are encoded by strings over $S_{\star} \times \Sigma$ of length $n+2$. A configuration $u s v$, where $u, v \in \Sigma^{*}$ and $s \in S$, is written as $\langle\star, v(k)\rangle\langle\star, v(k-1)\rangle \cdots\langle\star, v(2)\rangle\langle s, v(1)\rangle\langle\star, u(m)\rangle \cdots\langle\star, u(1)\rangle$ where $k$ and $m$ are the lengths of $v$ and $u$, resp., and $v(i)$ denotes the $i^{\text {th }}$ symbol of $v$ (configurations are represented in a 'reversed order' since we want to write the top stack symbol on the left-hand side). Elements of $S \times \Sigma_{\star} \times\{0, \cdots, n+2\}$ are used as top stack symbols when pushing a new configuration to the stack (see below); they should 
be seen as a finite memory where we keep (and update) the information about the position of the symbol which will be guessed by the next transition (as we count symbols from zero, the bounded counter reaches the value $n+2$ after guessing the last symbol), about the control state which is to be pushed, and about the (only) symbol of the form $\langle s, a\rangle$ which was actually pushed. The $Z$ is a special 'bottom' symbol which can emit all actions and cannot be popped. The role of symbols of $S \times \Sigma \times\{W\} \cup\{T\}$ will be clarified later. The set of actions is $A c t=\{a, c, f, s, d, t\} \cup\left(S_{\star} \times \Sigma\right)$, and $\delta$ consists of the following transitions:

1. $(\langle s, \star\rangle, i) \stackrel{a}{\rightarrow}(\langle s, \star\rangle, i+1)\langle\star, A\rangle$

2. $(\langle s, \star\rangle, i) \stackrel{a}{\rightarrow}(\langle s, A\rangle, i+1)\langle s, A\rangle$

3. $(\langle s, A\rangle, i) \stackrel{a}{\rightarrow}(\langle s, A\rangle, i+1)\langle\star, B\rangle$

4. $(\langle s, A\rangle, n+2) \stackrel{c}{\rightarrow}(\langle s, A\rangle, W)$

5. $(\langle s, A\rangle, W) \stackrel{d}{\rightarrow} \varepsilon$

6. $(\langle s, A\rangle, W) \stackrel{f}{\rightarrow}\left(\left\langle s^{\prime}, \star\right\rangle, 0\right)$

7. $(\langle s, A\rangle, W) \stackrel{s}{\rightarrow}\left(\left\langle s^{\prime}, \star\right\rangle, 0\right)$

8. $(\langle s, A\rangle, W) \stackrel{f}{\rightarrow}\left(\left\langle s^{\prime}, \star\right\rangle, 0\right)$

9. $(\langle s, A\rangle, W) \stackrel{s}{\rightarrow}\left(\left\langle s^{\prime}, \star\right\rangle, 0\right)$

10. $(\langle s, A\rangle, W) \stackrel{y}{\rightarrow} T$

11. $T \stackrel{t}{\stackrel{t}{\longrightarrow}} T$

12. $Z \stackrel{y}{\rightarrow} Z$

13. $\langle x, A\rangle \stackrel{\langle x, A\rangle}{\rightarrow} \varepsilon$ for all $A \in \Sigma, s \in S, 0 \leq i \leq n+1$; for all $A \in \Sigma, s \in S, 0 \leq i \leq n+1$; for all $A, B \in \Sigma, s \in S, 0 \leq i \leq n+1$; for all $A \in \Sigma, s \in S$; for all $s \in S, A \in \Sigma$ such that $s$ is not rejecting;

for all $s, s^{\prime} \in S, A \in \Sigma$ such that $\pi(s) \in\{\forall, \exists\}$ and $s^{\prime}=\operatorname{first}(s, A)$;

for all $s, s^{\prime} \in S, A \in \Sigma$ such that $\pi(s) \in\{\forall, \exists\}$ and $s^{\prime}=\operatorname{second}(s, A)$;

for all $s, s^{\prime} \in S, A \in \Sigma$ such that $\pi(s)=\exists$ and $s^{\prime}=\operatorname{second}(s, A)$;

for all $s, s^{\prime} \in S, A \in \Sigma$ such that $\pi(s)=\exists$ and $s^{\prime}=\operatorname{first}(s, A)$;

for all $s \in S, y \in\{f, s\}$ such that $\pi(s)=a c c$;

for all $y \in A c t$;

for all $x \in S_{\star}, A \in \Sigma$.

The process $\alpha$ corresponds to the initial configuration of $\mathcal{M}$, i.e.,

$$
\alpha=\left(\left\langle s_{0}, \vdash\right\rangle, n+2\right)\langle\star,-1\rangle\langle\star, w(n)\rangle \cdots\langle\star, w(2)\rangle\langle\star, w(1)\rangle\left\langle s_{0}, \vdash\right\rangle Z
$$

The behavior of $\alpha$ can be described as follows: whenever the top stack symbol is of the form $(\langle s, A\rangle, W)$, we know that the previously pushed configuration contains the symbol $\langle s, A\rangle$. If $s$ is rejecting, no further transitions are possible. Otherwise, $(\langle s, A\rangle, W)$ can either disappear (emitting the action $d$-see rule 5), or it can perform one of the $f$ and $s$ actions as follows:

- If $s$ is universal or existential, $(\langle s, A\rangle, W)$ can emit either $f$ or $s, \operatorname{storing} f \operatorname{frst}(s, A)$ or $\operatorname{second}(s, A)$ in the top stack symbol, respectively (rules 6,7$)$.

- If $s$ is existential, $(\langle s, A\rangle, W)$ can also emit $f$ and $s$ while storing $\operatorname{second}(s, A)$ and $\operatorname{first}(s, A)$, respectively (rules 8,9$)$.

- If $s$ is accepting, $(\langle s, A\rangle, W)$ emits $f$ or $s$ and pushes the symbol $T$ which can do the action $t$ forever (rules 10,11). 
If $(\langle s, A\rangle, W)$ disappears, the other symbols stored in the stack subsequently perform their symbol-specific actions and disappear (rule 13). If $s$ is not accepting and $(\langle s, A\rangle, W)$ emits $f$ or $s$, a new configuration is guessed and pushed to the stack; the construction of $\delta$ ensures that

- exactly $n+2$ symbols are pushed (rules $1-4$ );

- at most one symbol of the form $\left\langle s^{\prime}, B\right\rangle$ is pushed; moreover, the $s^{\prime}$ must be the control state stored in the top stack symbol. After pushing $\left\langle s^{\prime}, B\right\rangle$, the $B$ is also remembered in the top stack symbol (rule 2);

- if no symbol of the form $\left\langle s^{\prime}, B\right\rangle$ is pushed, no further transitions are possible after guessing the last symbol of the configuration (there are no transitions for symbols of the form $\left.\left(\left\langle s^{\prime}, *\right\rangle, n+2\right)\right)$;

- after pushing the last symbol, the action $c$ is emitted and a 'waiting' symbol $\left(\left\langle s^{\prime}, B\right\rangle, W\right)$ is pushed.

Now we define the finite-state system $\mathcal{F}$. The set of states of $\mathcal{F}$ is given by

$$
S=\left\{X, F, S, U, C_{0}, \cdots, C_{n}\right\} \cup\left\{C_{0}, \cdots, C_{n}\right\} \times\{0, \cdots, n+1\} \times\left(S_{\star} \times \Sigma\right)_{\star}^{4} .
$$

Transitions of $\mathcal{F}$ are

1. $X \stackrel{a}{\rightarrow} X, X \stackrel{c}{\rightarrow} F, X \stackrel{c}{\rightarrow} S, X \stackrel{c}{\rightarrow} C_{i} \quad$ for every $0 \leq i \leq n$;

2. $F \stackrel{f}{\rightarrow} X, F \stackrel{y}{\rightarrow} U \quad$ for every $y \in A c t-\{f\}$;

3. $S \stackrel{s}{\rightarrow} X, S \stackrel{y}{\rightarrow} U$

4. $C_{i} \stackrel{d}{\rightarrow}\left(C_{i}, 0, \star, \star, \star, \star\right), C_{i} \stackrel{y}{\rightarrow} U$ for every $y \in A c t-\{s\}$;

5. $U \stackrel{y}{\rightarrow} U$ for every $0 \leq i \leq n, y \in A c t-\{d\}$;

6. $\left(C_{i}, j, \star, \star, \star, \star\right) \stackrel{y}{\rightarrow}\left(C_{i}, j+1, \star, \star, \star, \star\right)$ for all $0 \leq i \leq n, 0 \leq j<i$, and $y \in$ $S_{\star} \times \Sigma$

7. $\left(C_{i}, i, \star, \star, \star, \star\right) \stackrel{y}{\rightarrow}\left(C_{i}, i+1, y, \star, \star, \star\right)$ for all $0 \leq i \leq n$ and $y \in S_{\star} \times \Sigma$;

8. $\left(C_{i}, i+1, y, \star, \star, \star\right) \stackrel{z}{\longrightarrow}\left(C_{i},(i+2) \bmod (n+2), y, z, \star, \star\right)$ for all $0 \leq i \leq n$ and $y, z \in S_{\star} \times \Sigma$;

9. $\left(C_{i}, j, y, z, \star, \star\right) \stackrel{u}{\rightarrow}\left(C_{i},(j+1) \bmod (n+2), y, z, \star, \star\right)$ for all $0 \leq i \leq n, i+2 \leq j \leq n+1$, and $y, z \in S_{\star} \times \Sigma$;

10. $\left(C_{i}, j, y, z, \star, \star\right) \stackrel{u}{\rightarrow}\left(C_{i}, j+1, y, z, \star, \star\right)$ for all $0 \leq i \leq n, 0 \leq j<i$, and $y, z, u \in S_{\star} \times \Sigma$;

11. $\left(C_{i}, i, y, z, \star, \star\right) \stackrel{u}{\longrightarrow}\left(C_{i}, i+1, y, z, u, \star\right)$ for all $0 \leq i \leq n$ and $y, z, u \in S_{\star} \times \Sigma$;

12. $\left(C_{i}, i+1, y, z, u, \star\right) \stackrel{v}{\rightarrow}\left(C_{i},(i+2) \bmod (n+2), y, z, u, v\right)$ for all $0 \leq i \leq n$ and $y, z, u, v \in S_{\star} \times \Sigma$;

13. $\left(C_{i},(i+2) \bmod (n+2), y, z, u, v\right) \stackrel{\stackrel{x}{\longrightarrow}}{\longrightarrow} U$ for all $0 \leq i \leq n, x \in A c t$, and $y, z, u, v \in S_{\star} \times \Sigma$ such that $(y, z)$ and $(u, v)$ are not compatible pairs (see below).

A fragment of $\mathcal{F}$ is shown in Fig. 1 . The role of states of the form $\left(C_{i}, 0, \star, \star, \star, \star\right)$ and their successors (which are not drawn in Fig. 1) is clarified below.

Now we prove that $\mathcal{M}$ accepts $w$ iff $\alpha \nsubseteq X$. Intuitively, the simulation game between $\alpha$ and $X$ corresponds to constructing a branch in a computational tree for $\mathcal{M}$ 

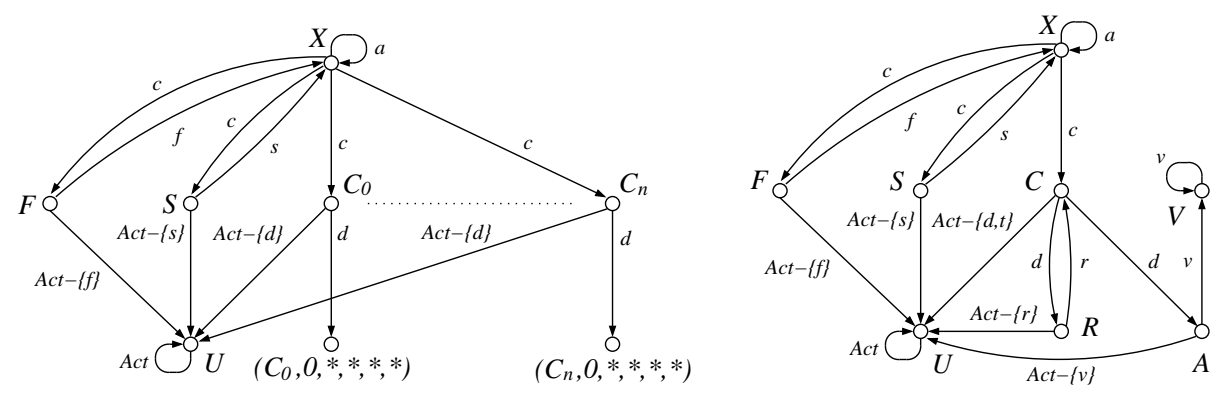

Fig. 1. The systems $\mathcal{F}$ and $\mathcal{F}^{\prime}$ (successors of $\left(C_{i}, 0, \star, \star, \star, \star\right)$ in $\mathcal{F}$ are omitted).

on $w$. The attacker (who plays with $\alpha$ ) wants to show that there is an accepting computational tree, while the defender aims to demonstrate the converse. The attacker is therefore 'responsible' for choosing the appropriate successors of all existential configurations (selecting those for which an accepting subtree exists), while the defender chooses successors of universal configurations (selecting those for which no accepting subtree exists). The attacker wins iff the constructed branch reaches an accepting configuration. The choice is implemented as follows: after pushing the last symbol of a configuration, the attacker has to emit the $c$ action and push a 'waiting' symbol (see above). The defender can reply by entering the state $F, S$, or one of the $C_{i}$ states. Intuitively, he chooses among the possibilities of selecting the first or the second successor, or checking that the $i^{t h}$ symbol of the lastly pushed configuration was guessed correctly (w.r.t. the previous configuration). Technically, the choice is done by forcing the attacker to emit a specific action in the next round-observe that if the defender performs, e.g., the $X \stackrel{c}{\rightarrow} F$, transition, then the attacker must use one of his $f$ transitions in the next round, because otherwise the defender would go immediately to the state $U$ where he can simulate 'everything', i.e., the attacker loses the game. As the defender is responsible only for selecting the successors of universal configurations, the attacker has to follow his 'dictate' only if the lastly pushed configuration was universal; if it was existential, he can choose the successor according to his own will (see the rules 6-9 in the definition of $\delta$ ). If the lastly pushed configuration was rejecting, the attacker cannot perform any further transitions from the waiting symbol, which means that the defender wins. If the configuration was accepting and the defender enters $F$ of $S$ via the action $c$, then the attacker wins; first he replaces the waiting symbol with $T$, emitting $f$ or $s$, resp. (so that the defender has to go back to $X$ ) and then he does the action $t$. The purpose of the states $C_{i}$ (and their successors) is to ensure that the attacker cannot gain anything by 'cheating', i.e., by guessing configurations incorrectly. If the defender is suspicious that the attacker has cheated when pushing the last configuration, he can 'punish' the attacker by going (via the action $c$ ) to one of the $C_{i}$ states. Doing so, he forces the attacker to remove the waiting symbol in the next round (see the rule 5 in the definition of $\delta$ ). Now the atacker can only pop symbols from the stack and emit the symbol-specific actions. The defender 'counts' those actions and 'remembers' the symbols at positions $i$ and $i+1$ in the lastly and the previously pushed configurations. After the defender collects the four symbols, he either enters a universal state $U$ (i.e., he wins the game), or gets 'stuck' (which means that the attacker wins). It depends on whether the two pairs 
of symbols are compatible w.r.t. the transition function $\gamma$ of $\mathcal{M}$ or not (here we use a folklore technique of checking the consistency of successive configurations of Turing machines). Observe that if the lastly pushed configuration was accepting, the defender still has a chance to perform a consistency check (in fact, it is his 'last chance' to win the game). On the other hand, if the defender decides to check the consistency right at the beginning of the game (when the attacker plays the $c$ transition from $\alpha$ ), he inevitably loses because the attacker reaches the bottom symbol $Z$ in $n+2$ transitions and then he can emit the action $t$. It follows that the attacker has a winning strategy iff $\mathcal{M}$ accepts $w$.

Theorem 2. The problem PDA $5 \boldsymbol{F S}$ is EXPTIME-hard even for a fixed finite-state process.

Proof. We modify the construction of Theorem 1. Intuitively, we just re-implement the cheating detection so that the compatibility of selected pairs of symbols is checked by the pushdown system and not by $\mathcal{F}$ (now we can store the four symbols in the finite control). However, it must still be the defender who selects the (position of the) pair. This can be achieved with a fixed number of states (see [5]).

Theorem 3. The problem $\boldsymbol{F S} \sqsubseteq \boldsymbol{B P A}$ is EXPTIME-hard.

Proof. The technique is similar to the one of Theorem 1 (see [5]).

Theorem 4. The problem $\boldsymbol{F S} \sqsubseteq \boldsymbol{P D A}$ is EXPTIME-hard even for a fixed finite-state process.

An immediate consequence of Theorem 1 and Theorem 2 is the following:

Corollary 5. The problem $\boldsymbol{B P A} \simeq \boldsymbol{F S}$ is EXPTIME-hard. Moreover, the problem $\boldsymbol{P D A} \simeq \boldsymbol{F S}$ is EXPTIME-hard even for a fixed finite-state process.

Proof. There is a simple (general) reduction from the $A \sqsubseteq B$ problem to the $A \simeq B$ problem (where $A, B$ are classes of processes) which applies also in this case-given processes $p \in A$ and $q \in B$, we construct processes $p^{\prime}, q^{\prime}$ such that $p^{\prime}$ has only the transitions $p^{\prime} \stackrel{a}{\rightarrow} p, p^{\prime} \stackrel{a}{\rightarrow} q$, and $q^{\prime}$ has only the transition $q^{\prime} \stackrel{a}{\rightarrow} q$. It follows immediately that $p^{\prime} \simeq q^{\prime}$ iff $p \sqsubseteq q$.

The problem of PDA $\sim$ PDA is decidable, but the exact complexity is not known. The decision procedures described in $[9,13]$ do not give any upper complexity bound. So far, the best known lower bound for this problem was PSPACE-hardness [8]. However, while the problem PDA $\sim$ FS is $P S P A C E$-complete (see Section 3) the problem PDA PDA is at least EXPTIME-hard. This EXPTIME lower bound even holds for the subclass of normed PDA (a PDA is normed iff from every reachable configuration it is possible to empty the stack).

The proof of the following theorem uses a technique which can be traced back to Jančar [1]; a more explicit formulation is due to Srba [10] who used the technique in the different context of Basic Parallel Processes. The main idea is that in a bisimulation game the defender can force the attacker to do certain things according to the defender's choices. The full proof can be found in [5]. 
Theorem 6. The problem PDA $\sim \boldsymbol{P D A}$ is EXPTIME-hard, even for normed PDA.

Proof. (sketch) The proof is done by a polynomial-time reduction of the (EXPTIMEcomplete) acceptance problem of alternating LBA to the PDA $\sim$ PDA problem. The bisimulation game proceeds as follows. The attacker guesses LBA configurations and pushes them onto the stack. The defender is forced to copy these moves. At existential control states (of the LBA) the attacker chooses the successor control state, and at the universal control states (of the LBA) the defender gets to choose the successor control state (this requires the technique mentioned above where the defender forces the attacker to do certain things). At any time, the defender can force the attacker to enter a so-called check-phase. In this check-phase it is verified if the LBA configuration at the top of the stack is really a successor configuration (according to the transition rules of the LBA) of the LBA configuration that was pushed onto the stack before. If not, then the defender wins the bisimulation game. This construction forces the attacker to play 'honestly', i.e., to correctly simulate the behavior of the LBA. If an accepting configuration (of the LBA) is reached in this way then the attacker wins the bisimulation game (having proved, despite the interference of the defender's choices at the universal control states, that the alternating LBA accepts). Otherwise, the bisimulation game goes on forever and the defender wins. This construction ensures that the attacker has a winning strategy if and only if the alternating LBA accepts. Thus, the alternating LBA accepts iff the two PDAs are not bisimilar.

\section{Upper Bounds}

The next theorem extends the result for strong simulation which appeared in [6]; the proof is based on the same idea, but the constructed formula $\varphi$ is now completely fixed.

Theorem 7. The problems $\boldsymbol{P D A} \sqsubseteq_{w} \boldsymbol{F S}, \boldsymbol{F S} \sqsubseteq_{w} \boldsymbol{P D A}$, and $\boldsymbol{P D A} \simeq_{w} \boldsymbol{F S}$ are in EXPTIME.

Proof. All of the above mentioned problems are polynomially reducible to the modelchecking problem with pushdown automata and a fixed formula $\varphi$ of the modal $\mu$ calculus (which is decidable in deterministic exponential time [17]).

Let $\varphi \equiv \nu X . \square_{a} \diamond_{b}\langle c\rangle X$, where $\square_{a} \psi \equiv \nu Y .(\psi \wedge[a] Y)$ and $\diamond_{b} \psi \equiv \nu Z .(\psi \vee\langle b\rangle Z)$. Intuitively, $\square_{a} \psi$ says that each state which is reachable from a given process via a finite sequence of $a$-transitions satisfies $\psi$, and $\diamond_{b} \psi$ says that a given process can reach a state satisfying $\psi$ via a finite sequence of $b$-transitions. Hence, the meaning of $\varphi$ can be explained as follows: a process satisfies $\varphi$ iff after each finite sequence of $a$-transitions it can perform a finite sequence of $b$-transitions ended with one $c$-transition so that the state which is entered again satisfies $\varphi$ (we refer to [3] for a precise definition of the syntax and semantics of the modal $\mu$-calculus). Now let $\Delta=(Q, \Gamma, A c t, \delta)$ be a pushdown system, $\mathcal{F}=(F, A c t, \rightarrow)$ a finite-state system, $p \alpha$ a process of $\Delta$, and $f$ a process of $\mathcal{F}$. We construct a pushdown system $\Delta=\left(Q \times F \times A c t \times\{0,1\}, \Gamma \cup\{Z\},\{a, b, c\}, \delta^{\prime}\right)$ (where $Z \notin \Gamma$ is a new bottom symbol) which 'alternates' the $\stackrel{x}{\Rightarrow}$ transitions of $\Delta$ and $\mathcal{F}$, remembering the ' $x$ ' in its finite control. Formally, $\delta$ ' is constructed as follows:

- for all $q A \stackrel{x}{\rightarrow} r \beta \in \delta$ and $g \in F$ we add $(q, g, \tau, 0) A \stackrel{a}{\rightarrow}(r, g, x, 0) \beta$ to $\delta^{\prime}$; 
- for all $q A \stackrel{\tau}{\rightarrow} r \beta \in \delta, x \in A c t$, and $g \in F$ we add $(q, g, x, 0) A \stackrel{a}{\rightarrow}(r, g, x, 0) \beta$ to $\delta^{\prime}$;

- for all $q \in Q, g \in F, x \in A c t$, and $Y \in \Gamma \cup\{Z\}$ we add $(q, g, x, 0) Y \stackrel{b}{\rightarrow}$ $(q, g, x, 1) Y$ to $\delta^{\prime}$;

- for each transition $g \stackrel{x}{\rightarrow} g^{\prime}$ of $\mathcal{F}$ and all $q \in Q, Y \in \Gamma \cup\{Z\}$ we add $(q, g, x, 1) Y \stackrel{b}{\rightarrow}$ $\left(q, g^{\prime}, \tau, 1\right) Y$ to $\delta^{\prime}$

- for all $g \stackrel{\tau}{\rightarrow} g^{\prime}$ of $\mathcal{F}, x \in A c t, q \in Q$, and $Y \in \Gamma \cup\{Z\}$ we add $(q, g, x, 1) Y \stackrel{b}{\rightarrow}$ $\left(q, g^{\prime}, x, 1\right) Y$ to $\delta^{\prime}$

- for all $q \in Q, g \in F$, and $Y \in \Gamma \cup\{Z\}$ we add $(q, g, \tau, 1) Y \stackrel{c}{\rightarrow}(q, g, \tau, 0) Y$ to $\delta^{\prime}$;

We claim that $p \alpha \sqsubseteq w f$ iff $(p, f, \tau, 0) \alpha Z \models \varphi$. Indeed, each sequence of $a$-transitions of $(p, f, \tau, 0) \alpha Z$ corresponds to some $\stackrel{x}{\Rightarrow}$ move of $p \alpha$ and vice versa; and after each such sequence, the 'token' can be switched from 0 to 1 (performing $b$ ), and now each sequence of $b$ 's ended with one $c$ corresponds to a $\stackrel{x}{\Rightarrow}$ move of $f$. Then, the token is switched back to 0 and the computation proceeds in the same way. $\varphi$ says that this can be repeated forever, unless we reach a state which cannot do any $a$ when the token is set to 0 . The new bottom symbol $Z$ has been added to ensure that $(p, f, \tau, 0) \alpha Z$ cannot get stuck just due to the emptiness of the stack. The FS $\sqsubseteq_{w}$ PDA direction is handled in a very similar way (the roles of $p \alpha$ and $f$ are just interchanged).

Corollary 8. The problems $\boldsymbol{B P A} \sqsubseteq_{w} \boldsymbol{F S}, \boldsymbol{F S} \sqsubseteq w \boldsymbol{B P A}$, and $\boldsymbol{B P A} \simeq_{w} \boldsymbol{F S}$ are decidable in polynomial time for (any) fixed finite-state process.

Proof. The complexity result of [17] says that model-checking with any fixed formula of the modal $\mu$-calculus and pushdown processes with a fixed number of control states is decidable in polynomial time. By synchronizing a given BPA process with a given (fixed) finite-state process as in Theorem 7 we obtain a pushdown system with a fixed number of control states, and the result follows.

Now we show that the problem PDA $\approx \mathbf{F S}$ is in PSPACE. First, we recall some results from [2]. A characteristic formula of a finite-state system $F$ w.r.t. $\approx$ is a formula $\Theta_{F}$ s.t. for every general system $G$ which uses the same set of actions as $F$ we have that $G \models \Theta_{F} \Longleftrightarrow G \approx F$. It has been shown in [2] that characteristic formulae for finitestate systems w.r.t. $\approx$ can be effectively constructed in the temporal logic EF (a simple fragment of CTL), by using the following theorem (here, $\approx_{k}$ denotes 'weak bisimilarity up-to $k$, which means that the defender has a strategy to defend for at least $k$ rounds in the weak bisimulation game).

Theorem 9. (taken from [2])

Let $F$ be a finite-state system with $n$ states and $G$ a general system. States $g \in G$ and $f \in F$ are weakly bisimilar iff the following conditions hold: (1) $g \approx_{n} f$ and (2) For each state $g^{\prime}$ which is reachable from $g$ there is a state $f^{\prime} \in F$ such that $g^{\prime} \approx_{n} f^{\prime}$.

One constructs characteristic formulae $\Phi_{k, f}$ for states $f$ in $F$ w.r.t. $\approx_{k}$ that satisfy $g \models$ $\Phi_{k, f} \Longleftrightarrow g \approx_{k} f$. The family of $\Phi_{k, f}$ formulae is defined inductively on $k$ as follows:

$$
\Phi_{0, f}:=\text { true }
$$




$$
\Phi_{k+1, f}:=\left(\bigwedge_{a \in A c t} \bigwedge_{f^{\prime} \in S(f, a)} \diamond_{a} \Phi_{k, f^{\prime}}\right) \wedge\left(\bigwedge_{a \in A c t}\left(\neg \diamond_{a}\left(\bigwedge_{f^{\prime} \in S(f, a)} \neg \Phi_{k, f^{\prime}}\right)\right)\right)
$$

where $S(f, a)=\left\{f^{\prime} \mid f \stackrel{a}{\rightarrow} f^{\prime}\right\}$ and $\diamond_{\tau}$ means "reachable via a finite number of $\tau$-transitions" and $\diamond_{a}:=\diamond_{\tau}\langle a\rangle \diamond_{\tau}$ for $a \neq \tau$.

Empty conjunctions are equivalent to true. Thus, by Theorem 9, the characteristic formula $\Theta_{f}$ for a process $f$ of a finite-state system $\mathcal{F}=(F$, Act,$\rightarrow)$ with $n$ states is

$$
\Theta_{f} \equiv \Phi_{n, f} \wedge \neg \diamond\left(\bigwedge_{f^{\prime} \in F} \neg \Phi_{n, f^{\prime}}\right)
$$

So one can reduce the problem $\mathbf{P D A} \approx \mathbf{F S}$ to a model checking problem for pushdown automata and (a slight extension of) the logic EF. The following proof-sketch uses many results by Walukiewicz [16]. For a complete proof, it would be necessary to repeat many of these, so we just sketch the main ideas and the crucial modification of the algorithm from [16]. It has been shown by Walukiewicz in [16] that model checking pushdown automata with the logic EF is PSPACE-complete. But our result does not follow directly from that. First, our characteristic formulae use a slight extension of EF, because of the $\diamond_{\tau}$ operator (normal EF has only the $\diamond$ operator). However, the model checking algorithm of [16] can trivially be generalized to this extension of EF, without increasing its complexity. The second, and more important problem is that the size of the characteristic formula $\Theta_{F}$ is exponential in $n$ (where $n$ is the number of states of $F$ ). However, a closer analysis of the model checking algorithm presented in [16] reveals that its complexity does not depend directly on the size of the formula, but rather on the number of its distinct subformulae. More precisely, this algorithm uses a so-called assumption function that assigns sets of subformulae to every control-state of the PDA. Of course, each EF formula has only a polynomial number of subformulae and hence the assumption function can be represented in polynomial space. However, it is also true for our characteristic formula $\Theta_{F}$ - although its size is exponential in $n$, the number of its distinct subformulae $\Phi_{k, f}$ is bounded by $\mathcal{O}\left(n^{2}\right)$, because $0 \leq k \leq n$ and $F$ has only $n$ states. Hence, we can run the mentioned model-checking algorithm for EF. Instead of 'unwinding' the $\Phi_{k, f}$ subformulae, we keep the abbreviations $\Phi_{k, f}$ as long as possible and expand them only (on-the-fly) when necessary (using the inductive definitions above). Thus, the whole algorithm works in polynomial space and we obtain the following theorem.

Theorem 10. The problem PDA $\approx \boldsymbol{F S}$ is in PSPACE.

\section{Conclusions}

The following table summarizes the complexity of all problems of comparing PDA and BPA to finite-state systems w.r.t. strong and weak simulation preorder/equivalence and strong and weak bisimilarity. FS means a finite-state system that is part of the input of the problem, while $\mathcal{F}$ means "any fixed finite-state system" for the upper complexity bounds and "some fixed finite-state system" for the lower complexity bounds. 


\begin{tabular}{l|c|c|c|c|c|c|c|c|c|} 
& $\begin{array}{c}\sqsubseteq \text { FS } \\
\sqsubseteq \text { FS }\end{array}$ & $\begin{array}{c}\sqsubseteq \mathcal{F} \\
\sqsubseteq \mathcal{F}\end{array}$ & $\begin{array}{c}\mathbf{F S} \sqsubseteq w \\
\mathbf{F S} \sqsubseteq\end{array}$ & $\begin{array}{c}\mathcal{F} \sqsubseteq w \\
\mathcal{F} \sqsubseteq\end{array}$ & $\begin{array}{c}\simeq \text { FS } \\
\simeq \text { FS }\end{array}$ & $\begin{array}{c}\simeq \mathcal{F} \\
\simeq \mathcal{F}\end{array}$ & $\begin{array}{c}\approx \text { FS } \\
\sim \text { FS }\end{array}$ & $\sim \mathcal{F}$ & $\approx \mathcal{F}$ \\
\hline BPA & $\begin{array}{c}\text { EXPTIME } \\
\text { complete }\end{array}$ & in P & $\begin{array}{c}\text { EXPTIME } \\
\text { complete }\end{array}$ & in P & $\begin{array}{c}\text { EXPTIME } \\
\text { complete }\end{array}$ & in P & in P & in P & in P \\
\hline PDA & $\begin{array}{c}\text { EXPTIME } \\
\text { complete }\end{array}$ & $\begin{array}{c}\text { EXPTIME } \\
\text { complete }\end{array}$ & $\begin{array}{c}\text { EXPTIME } \\
\text { complete }\end{array}$ & $\begin{array}{c}\text { EXPTIME } \\
\text { complete }\end{array}$ & $\begin{array}{c}\text { EXPTIME } \\
\text { complete }\end{array}$ & $\begin{array}{c}\text { EXPTIME } \\
\text { complete }\end{array}$ & $\begin{array}{c}\text { PSPACE } \\
\text { complete }\end{array}$ & in P & $\begin{array}{c}\text { PSPACE } \\
\text { complete }\end{array}$ \\
\hline
\end{tabular}

Finally, we have also shown (in Theorem 6) that the problem PDA $\sim$ PDA of checking bisimilarity of two pushdown systems is EXPTIME-hard. Thus, it is harder than the problem PDA $\sim$ FS of checking bisimilarity of a pushdown system and a finite-state system, which is only PSPACE-complete.

\section{References}

[1] P. Jančar. High undecidability of weak bisimilarity for Petri nets. In Proceedings of CAAP'95, volume 915 of LNCS, pages 349-363. Springer, 1995.

[2] P. Jančar, A. Kučera, and R. Mayr. Deciding bisimulation-like equivalences with finite-state processes. Theoretical Computer Science, 258(1-2):409-433, 2001.

[3] D. Kozen. Results on the propositional $\mu$-calculus. Theoretical Computer Science, 27:333354, 1983.

[4] A. Kučera. On simulation-checking with sequential systems. In Proceedings of ASIAN 2000, volume 1961 of $L N C S$, pages 133-148. Springer, 2000.

[5] A. Kučera and R. Mayr. On the complexity of semantic equivalences for pushdown automata and BPA. Technical report FIMU-RS-2002-01, Faculty of Informatics, Masaryk University, 2002.

[6] A. Kučera and R. Mayr. Simulation preorder over simple process algebras. Information and Computation, 173(2):184-198, 2002.

[7] A. Kučera and R. Mayr. Weak bisimilarity between finite-state systems and BPA or normed BPP is decidable in polynomial time. Theoretical Computer Science, 270(1-2):677-700, 2002.

[8] R. Mayr. On the complexity of bisimulation problems for pushdown automata. In Proceedings of IFIP TCS'2000, volume 1872 of LNCS, pages 474-488. Springer, 2000.

[9] G. Sénizergues. Decidability of bisimulation equivalence for equational graphs of finite outdegree. In Proceedings of 39th Annual Symposium on Foundations of Computer Science, pages 120-129. IEEE Computer Society Press, 1998.

[10] J. Srba. Strong bisimilarity and regularity of basic parallel processes is PSPACE-hard. In Proceedings of STACS 2002, volume 2285 of LNCS, pages 535-546. Springer, 2002.

[11] B. Steffen and A. Ingólfsdóttir. Characteristic formulae for processes with divergence. Information and Computation, 110(1):149-163, 1994.

[12] C. Stirling. The joys of bisimulation. In Proceedings of MFCS'98, volume 1450 of LNCS, pages 142-151. Springer, 1998.

[13] C. Stirling. Decidability of DPDA equivalence. Theoretical Computer Science, 255:1-31, 2001.

[14] W. Thomas. On the ehrenfeucht-fraïssé game in theoretical computer science. In Proceedings of TAPSOFT'93, volume 668 of LNCS, pages 559-568. Springer, 1993.

[15] R. van Glabbeek. The linear time-branching time spectrum. Handbook of Process Algebra, pages 3-99, 1999.

[16] I. Walukiewicz. Model checking CTL properties of pushdown systems. In Proceedings of FST\&TCS 2000, volume 1974 of LNCS, pages 127-138. Springer, 2000.

[17] I. Walukiewicz. Pushdown processes: Games and model-checking. Information and Computation, 164(2):234-263, 2001. 